\title{
Filter-Bank-Based Narrowband Interference Detection and Suppression in Spread Spectrum Systems
}

\author{
Tobias Hidalgo Stitz \\ Institute of Communications Engineering, Tampere University of Technology, P.O. Box 553, 33101 Tampere, Finland \\ Email: tobias.hidalgo@tut.fi \\ Markku Renfors \\ Institute of Communications Engineering, Tampere University of Technology, P.O. Box 553, 33101 Tampere, Finland \\ Email: markku.renfors@tut.fi
}

Received 29 October 2002; Revised 27 October 2003; Recommended for Publication by Xiang-Gen Xia

\begin{abstract}
A filter-bank-based narrowband interference detection and suppression method is developed and its performance is studied in a spread spectrum system. The use of an efficient, complex, critically decimated perfect reconstruction filter bank with a highly selective subband filter prototype, in combination with a newly developed excision algorithm, offers a solution with efficient implementation and performance close to the theoretical limit derived as a function of the filter bank stopband attenuation. Also methods to cope with the transient effects in case of frequency hopping interference are developed and the resulting performance shows only minor degradation in comparison to the stationary case.
\end{abstract}

Keywords and phrases: narrowband interference cancellation, complex PR filter banks, DS-SS.

\section{INTRODUCTION}

Direct sequence spread spectrum (DS-SS) systems have several applications, for example, CDMA communications and advantages, low power spectral density, privacy of the communications, and an inherent immunity to narrowband interferences, due to the processing gain [1]. Nevertheless, this immunity is only effective up to certain interference power, making it necessary to apply additional techniques to suppress the effect of strong narrowband interferences if a degradation of the performance is to be avoided. Several interference suppression techniques have been proposed to process the signal in the time domain (e.g., adaptive transversal filtering) $[2,3,4,5,6]$ and in the transform domain $[2,7,8,9]$. These techniques take advantage of the knowledge of the wide spectral shape of the desired signal's spectrum as compared to the interferer's narrow spectrum. There are also methods in the spatial domain, using techniques like antenna diversity or beamforming $[2,10]$, although these methods are not exclusive to narrowband interference on a wideband SS signal. There exist more advanced approaches in the time-frequency domain $[11,12]$, like wavelet transformation-based methods. In situations in which the interfering environment changes quickly, time-domain techniques are too slow to work correctly. In these cases, frequency-domain techniques like the
FFT-based or the filter-bank-based methods perform better $[8,12]$.

The purpose of this paper is to analyse the performance of a filter-bank-based interference suppression system. To eliminate the effects of narrowband interference, a perfect reconstruction (PR) filter bank with interference detection and subband suppression logic is used. Efficient implementation of the complex, critically sampled filter bank is based on an extension of the extended lapped transform (ELT) to the complex case. Based on detection and excision algorithms that can be found in the literature [8], a new, improved recursive algorithm is developed. Simulations have been run with different types of narrowband interference sources (jammers), demonstrating promising performance even in high jammer power cases.

The structure of the paper is as follows. Section 2 introduces the idea of filter-bank-based interference suppression and proposes an efficient implementation for the filter bank. Also the novel excision algorithm is presented and methods to relieve the transient effects in case of frequency hopping interference are introduced. Section 3 presents a theoretical performance analysis of the system. In Section 4 a detailed system model is presented and performance simulation results are shown and discussed in Section 5. Finally, Section 6 summarizes the conclusions obtained from this research work. 


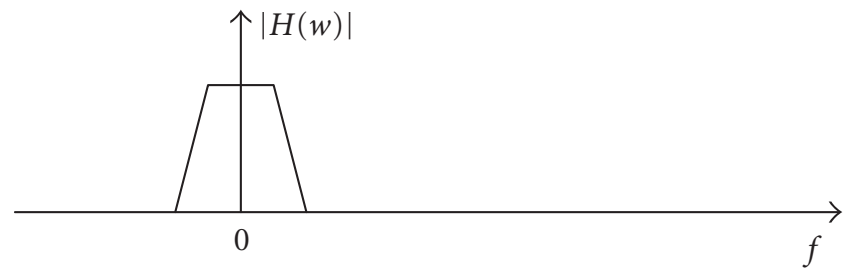

(a)

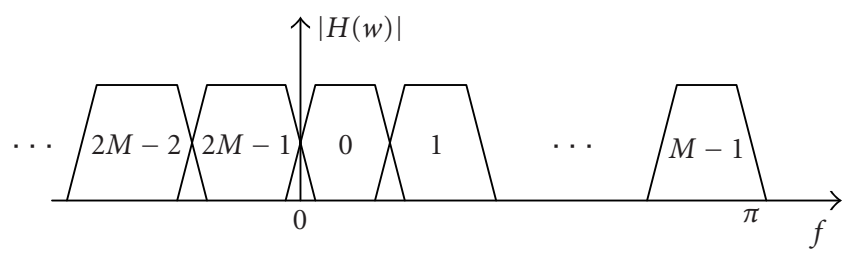

(b)

Figure 1: Modulated filter bank. (a) Prototype filter. (b) Complex modulated subband filters.

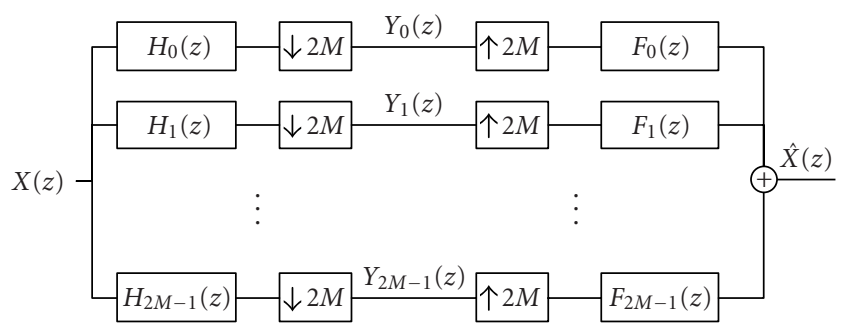

FIGURE 2: Maximally decimated 2M-channel analysis-synthesis filter bank system.

\section{FILTER BANKS FOR INTERFERENCE SUPPRESSION}

\subsection{Detection and suppression principle}

The interference suppressor presented in this paper is based on a complex modulated filter bank (MFB). In a complex MFB, a prototype filter $h_{p}(n)$ is modulated by a complex exponential function to yield $2 M$ bandpass filters in the form

$$
h_{k}(n)=h_{p}(n) \cdot e^{j n(2 k+1) \pi / 2 M} .
$$

As shown in Figure 1, the whole sampled frequency range can be divided into subbands by lining up consecutive filters. In the following, the terms "subchannel" and "subband" will be used interchangeably.

MFBs can be used to form analysis-synthesis filter banks that divide the received signal into several subchannels (analysis part), and reconstruct the original signal from the subchannels (synthesis part), after some optional processing. One benefit of this type of filter banks is that the processing can be done at a lower sampling rate, taking advantage

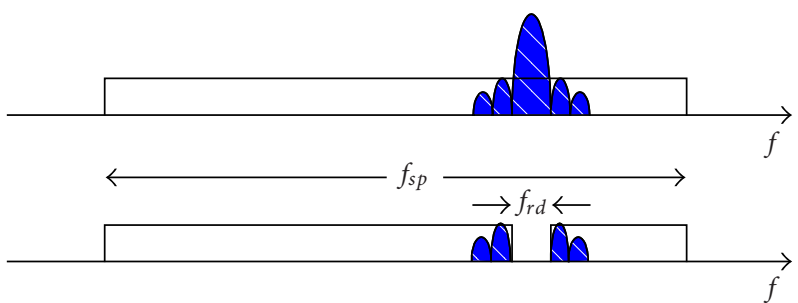

Figure 3: Application of the filter bank to the elimination of narrowband interference.

of the reduced bandwidth due to the subband filtering [13]. Figure 2 presents a maximally decimated filter bank, which means that, if the filter bank consists of $2 M$ channels, the factor by which the down- and upsampling is performed is also $2 M$.

If the filter design parameters are chosen correctly, the filter bank can offer PR, meaning that the output signal is just a scaled and delayed version of the input signal [13]:

$$
\hat{x}(n)=c x\left(n-n_{0}\right) .
$$

Applying the filter banks to the narrowband interference suppression problem, the subbands affected by the interference are not included in the synthesis part of the filter, resulting in notch filtering, as sketched in Figure $3[2,12]$.

Since the FFT can also be regarded as a filter bank, it can be used as an approach to remove the interference. However, each subchannel of the FFT filter bank has strong sidelobes, the first ones at $-13 \mathrm{~dB}$. Thus, the power of the narrowband jammer is very likely to leak to adjacent subchannels, affecting a relatively high portion of the signal bandwidth. To fight this limitation, windowing can be applied to the signal before taking the FFT [14]. Although this is an effective solution to lower the sidelobes, the conditions for sufficient aliasing cancellation in an efficient, maximally decimated system are not so well understood as in PR filter bank systems. It should also be emphasized that a straightforward application of the complex modulation principle does not provide a PR system in the maximally decimated case [15]. Our implementation of the filter bank overcomes these problems by using the novel maximally decimated filter bank structure [16] shown in Figure 4, based on the ELT [17]. Using a PR filter bank, we also assure that the signal does not suffer any additional distortion by the processing. This is especially important in the case in which there is no interference present and no subband processing takes place. Further, the PR filter banks have efficient implementation methods based on ELT that are not applicable in the non-PR case [18]. However, properly designed nearly-perfect-reconstruction (NPR) CMFB/SMFB filter banks can be used in the same configuration (Figure 4) to implement a complex NPR filter bank suitable for our application. Depending on the used hardware architecture and allowed performance degradation in the interference free case, such a design could be slightly more efficient than the PR bank. 


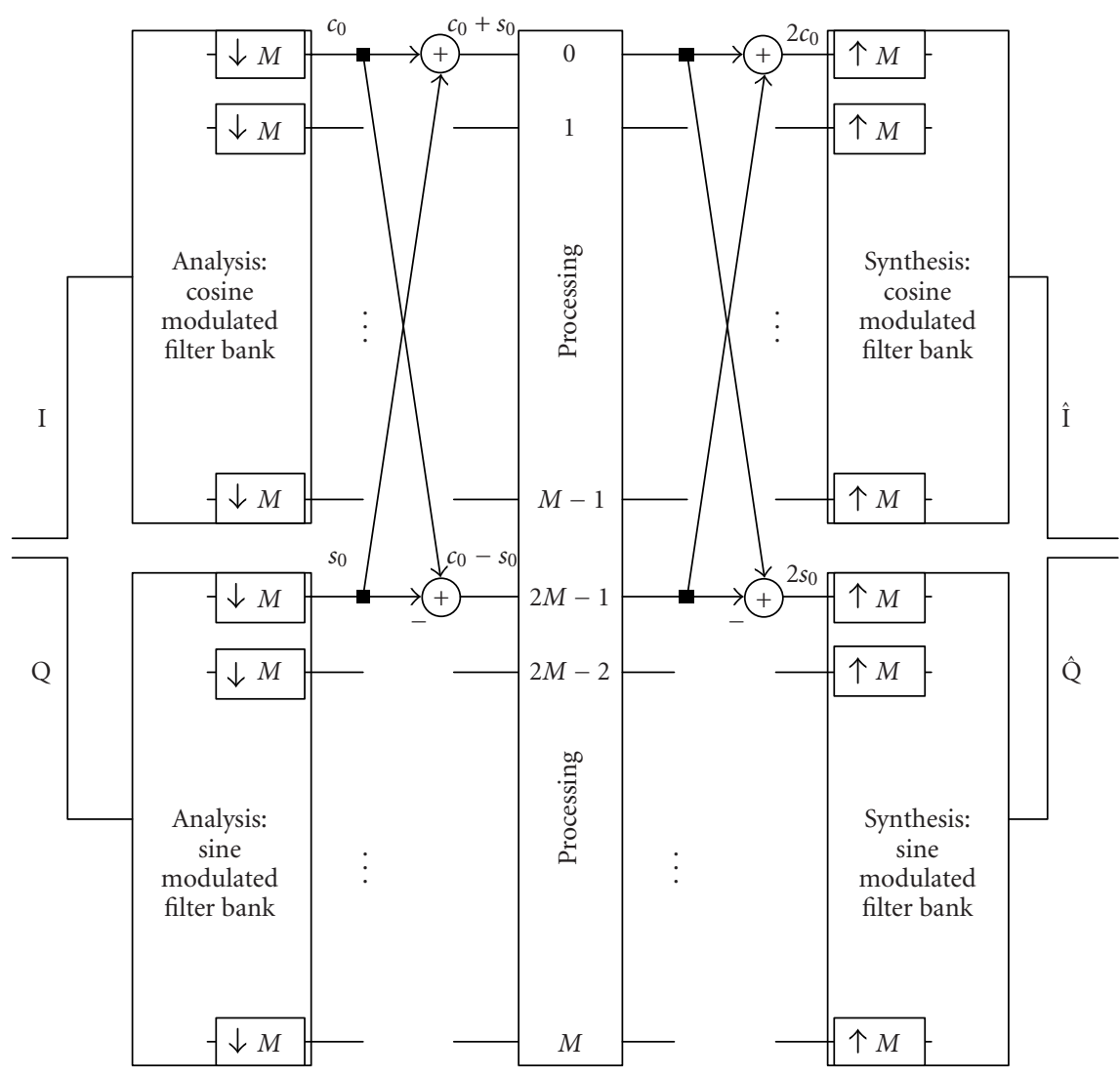

FIGURE 4: Realisation of the 2M-channel complex MFB using sine and cosine MFBs that can be implemented with real-valued ELTs.

\subsection{Complex critically sampled $P R$ filter bank structure}

Some papers have appeared proposing complex modulated lapped transforms for different applications, such as audio processing [19] and image motion estimation [20]. However, these papers use real-valued input signals to which the transform is applied.

The method chosen here to implement the complex MFB for the interference detection and suppression system with complex (I/Q) input signal is illustrated in Figure 4. The inputs are the real (I, in-phase) and imaginary $(\mathrm{Q}$, quadrature) parts of the complex signals. The PR cosine and sine MFBs and the following butterfly structures effectively allow obtaining real subband signals by separating the positive and negative parts of the spectrum corresponding to each real subband, as sketched in Figure 5. If we observe the subsampled signals of Figure 4, we can see that, due to the butterfly structures, at the entrance of the synthesis banks we have, for the $k$ th subchannel in the upper branch (CMFB),

$$
\left(c_{k}+s_{k}\right)+\left(c_{k}-s_{k}\right)=2 c_{k},
$$

and, in the lower branch (SMFB),

$$
\left(c_{k}+s_{k}\right)-\left(c_{k}-s_{k}\right)=2 s_{k},
$$

where $c_{k}$ and $s_{k}$ are the outputs of the analysis CMFB and SMFB for subchannel $k$, respectively (time index omitted). From the point of view of the filter bank, the signal remains unchanged (except for a scaling factor of 2), but in between the butterflies, the positive and negative sides of the spectra of the original signal at the entrance of the filter bank are separated. We can see this process in Figure 5. At the beginning we have a complex signal with different positive and negative frequency spectra. A certain spectral region and its corresponding symmetric negative counterpart are highlighted for better understanding. Next, the real and imaginary parts of the complex signal are separated and their corresponding spectra are shown. We then apply the filter bank and follow the changes suffered by the highlighted sections of the spectrum, sections that for simplicity coincide with the $k$ th subchannel of the filter bank. The real part of the signal is filtered by the CMFB and the imaginary by the SMFB and then decimated and combined by the first butterfly. This is reflected in the spectra of Figure 5 by the spectral expansion inherent to decimation and by the fact that in the upper branch $\left(c_{k}+s_{k}\right)$ we now have a signal corresponding to the positive side of the filtered spectrum section of the original signal $x$. In a same manner, the lower branch $\left(c_{k}-s_{k}\right)$ carries a signal corresponding to the negative side of the filtered spectrum section of the original signal $x$. Ignoring the processing stage, 


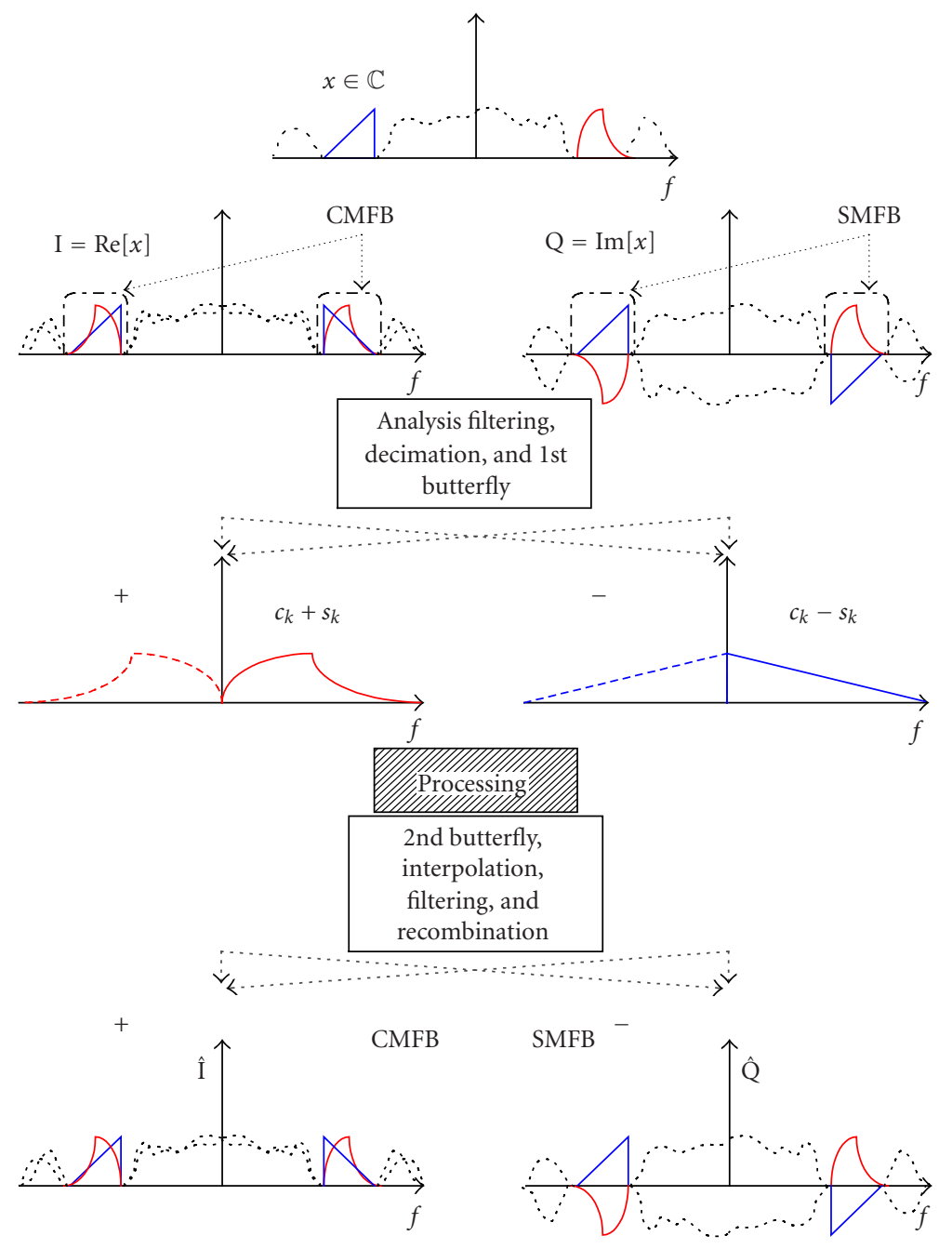

FIgURE 5: Separating and combining the spectral components using the structure of Figure 4.

after the second butterfly we recover the signals as they were before the first one (except for the scaling factor). Upsampling (spectral contraction) and recombining with the other subband signals yields Î and $\hat{Q}$, similar to I and Q, assuming there was no further processing of the signals involved.

In other words, the analysis subchannel filtering function is equivalent to applying the corresponding complex subband filter of the complex MFB to the input signal, taking the real part of the output and decimating by $M$. Thus, the subband signals of the basically complex bank are used in real format at the processing stage. Finally, after the synthesis bank, the filtered in-phase and quadrature high-rate signals are obtained, with perfect reconstruction if no processing has taken place.

In the proposed structure, all the operations at the processing stage take place with real instead of complex signals and arithmetic. In fact, it can be shown that perfect reconstruction can be achieved in a critically sampled system only if the subsampled signals are real. If the subsampled signals are complex, the necessary aliasing cancellation for achieving PR cannot be obtained [16].

The implementation of the cosine and sine MFBs with efficient algorithms has been well studied; lattice, polyphase, and ELT structures can be used [17, 21]. We use the ELTbased approach, based on DCT-IV and DST-IV, leading to an efficient implementation of the filter bank. In [16], it is shown that a complex PR system can be obtained from an ELT-based real PR system using the proposed approach.

\subsection{The excision principle}

The detection of the jammer is based on thresholds, taking into consideration the uniform shape of the DS-SS signal spectrum. Different adaptive threshold calculation methods for FFT-based systems have been studied and presented in [8]. The simplest effective one measures the powers of the subbands, obtains a mean of them, and multiplies it by a factor $t_{f}$ (threshold factor, $t_{f}>1$ ) to set up the threshold $\theta$. If 
we define the signals after the first butterflies as

$$
b_{k}= \begin{cases}c_{k}+s_{k}, & k=0, \ldots, M-1, \\ c_{2 M-1-k}-s_{2 M-1-k}, & k=M, \ldots, 2 M-1,\end{cases}
$$

we can write

$$
\theta=\frac{t_{f}}{2 M} \sum_{k=0}^{2 M-1} E\left(b_{k}^{2}\right)
$$

considering that the signals $b_{k}$ have zero mean. The subbands with higher powers are eliminated, so after the processing,

$$
\hat{b}_{k}= \begin{cases}b_{k}, & E\left(b_{k}^{2}\right)<\theta, \\ 0, & E\left(b_{k}^{2}\right) \geq \theta .\end{cases}
$$

However, there might be some jammer energy present in the neighbouring subchannels that is not detected in the first sweep, so our algorithm was built out to be recursive, as sketched in Figure 6. A similar excision algorithm has been developed independently in [22].

Once the subbands with detected jammer presence are removed, the same process is repeated without the removed subchannels. This can be described by rewriting (6) into the form

$$
\theta=\frac{t_{f}}{M_{r}} \sum_{\substack{k=0 \\ k \notin R}}^{2 M-1} \operatorname{var}\left(b_{k}\right),
$$

where $M_{r}$ is the number of active subbands that has not been set to 0 and $R$ represents the indices of the removed subbands. Thus, the algorithm checks if there are subchannels that could have passed the previous threshold but could still be affected by jammer power and exceed a newly set averaging threshold. This can happen if the jammer is very powerful, pulling the threshold up in such a way that the leaking to the neighbouring subbands is not detected in the first sweep. A possible further jammer with lower power would also not be detected. Setting a too low threshold factor to accelerate or even avoid the recursive algorithm could be counterproductive, especially if we have few signal samples at our disposal to calculate the subband signal power. In this case, there might be great variations in the subband power estimates, which would lead to wrong decisions if the threshold is low.

\subsection{Mitigating transient effects in case of frequency hopping interference}

We here consider mostly the case where the interference frequency may be changing or the interference may appear or disappear instantaneously. In such a case, it is natural to use relatively short processing blocks, the length of which is an integer multiple of the symbol interval. In this situation, one important source of errors is the transients that appear at the beginning and at the end of the processing blocks. In our work, different methods have been tried to mitigate the transient effects. The most effective one from the performance point of view is the use of a guard symbol at the end of the

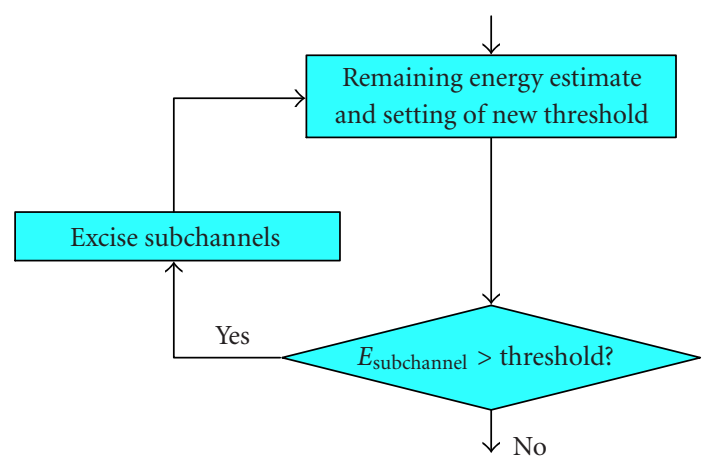

FIGURE 6: Recursive jammer detection principle.

block, where the transients cause more errors. Nevertheless, there is a more efficient way to fight the transients and to save the last bit for information: the guard interval. In this approach, the despreading of the last bit does not happen with the whole spreading code, only the last chips are discarded because of their distorted values due to the transients.

\section{PERFORMANCE ANALYSIS}

In a BPSK system with AWGN channel, the bit error rate (BER), as a function of the energy per bit to noise power spectral density ratio, can be estimated with the help of the Q-function as follows [1]:

$$
p_{b}=\mathrm{BER}=Q\left(\sqrt{2 \frac{E_{b}}{N_{0}}}\right) .
$$

In the case of a spread spectrum communication system with interference present in the channel, the BER can be estimated as

$$
\mathrm{BER}=Q\left(\sqrt{\frac{2 g_{p} S}{J+N}}\right)=Q\left(\sqrt{\frac{2 g_{p}(S / N)(S / J)}{S / N+S / J}}\right),
$$

where $g_{p}$ is the processing gain introduced by the despreading of the signal, and $S, J$, and $N$ are the powers of the signal, the jammer, and the noise, respectively. The quotient $S / N$ can be calculated from the energy per bit to noise power spectral density ratio as

$$
\frac{S}{N}=\frac{1}{g_{p}} \frac{E_{b}}{N_{0}} .
$$

To estimate the effect of removing some of the filter bank subbands of a BPSK signal, $E_{b} / N_{0}$ should be reduced by the factor $\left(f_{s p}-f_{r d}\right) / f_{s p}$ (see Figure 3) [8]. Thus, the effective $S / N$ becomes

$$
\frac{S}{N}=\frac{1}{g_{p}} \frac{E_{b}}{N_{0}} \frac{f_{s p}-f_{r d}}{f_{s p}} .
$$

Here, $f_{s p}$ is the bandwidth of the spread signal and $f_{r d}$ is the part of it that is being removed. It is assumed that the remaining jammer power is clearly below the noise power level. 


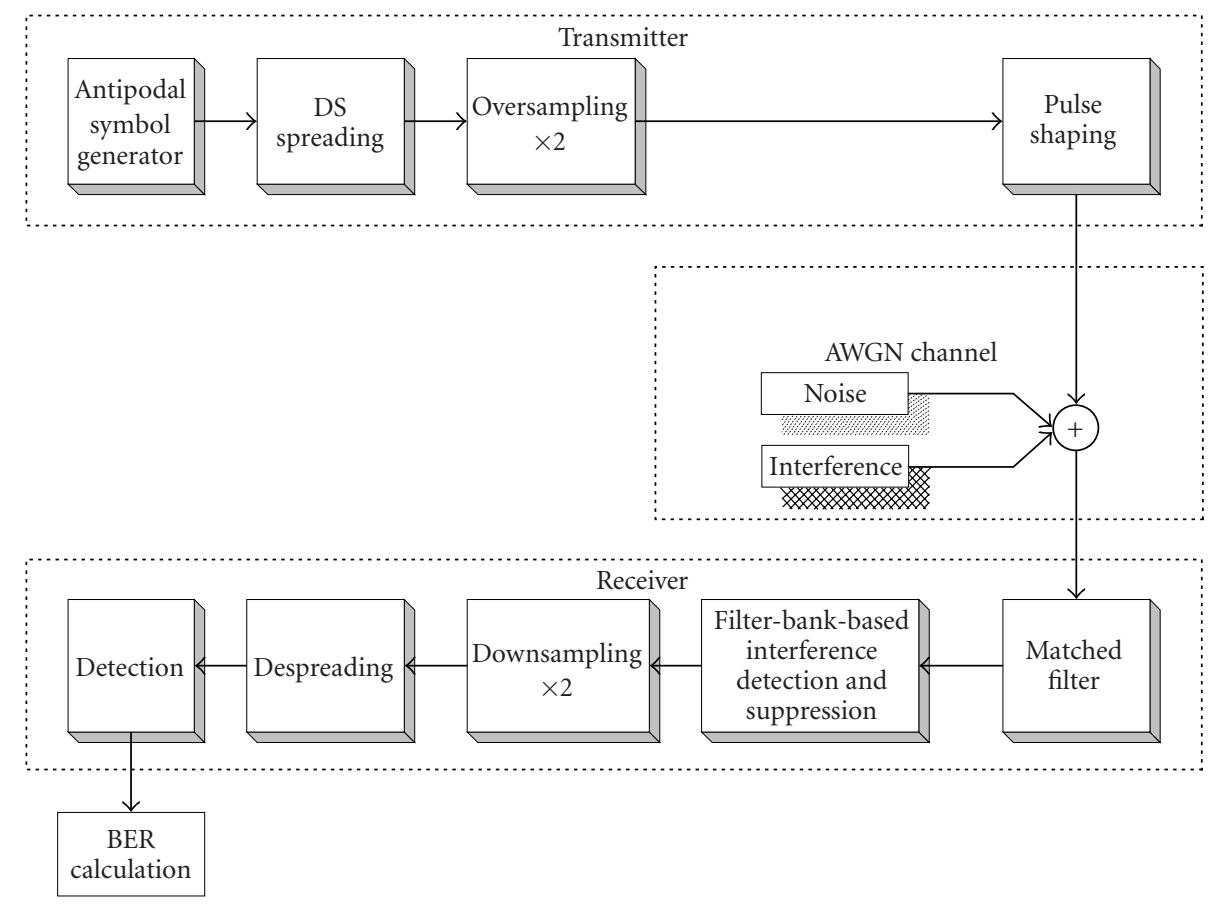

FIGURE 7: Block diagram of the general baseband system model.

Equation (12) permits to predict the expected performance if the bandwidth that is removed to fight the jammer is known. The bandwidth to be removed can be estimated from the bandwidth and power of the jammer and from the spectral characteristics of the prototype filter in the filter bank, since its stopband edge and attenuation determine how much jammer power can leak to neighbouring subchannels.

\section{SYSTEM MODEL}

The system used to model the narrowband interference suppressor is presented in Figure 7.

The figure shows a transmitter that sends information through a channel to a receiver, modelled in the baseband domain. At the transmitter, the antipodal signal generator generates a random sequence of 1's and -1 's. The generated binary sequence is spread by a pseudorandom $m$-sequence by multiplying each information bit by this sequence in the DS spreading block. Next, the sampling frequency is doubled and the obtained signal is filtered by a pulse shaping filter of the root-raised cosine type with a roll of factor of $22 \%$.

The channel is an AWGN channel with additive interference. The signals that model the noise and the interference are both complex. The jammer is either a single tone or a $10 \%$ BPSK-type interferer, pulse shaped with a roll of factor of $35 \%$. It occupies $10 \%$ of the desired signals bandwidth and can have either a fixed spectral position or hops in the range $\left[-f_{s} / 2, f_{s} / 2\right]$, where $f_{s}$ is the sampling rate, at regular intervals.

At the receiver, the signal is filtered by a digital matched filter at twice the chip rate. The interference detection and suppression block performs an estimation of the jammer location in the frequency axis and suppresses the bands that contain it. To achieve this goal, an ELT-based filter bank is used, dividing, respectively, the real part and the imaginary part of the received signal among $2 M$ real subbands.

Next, the inverse operations to the ones performed at the transmitter are completed: the jammer-free signal is downsampled and despread following the integrate-and-dump principle. Ideal code synchronization is assumed. The received signal is converted to a sequence of bits after the decisions have been made at the detector. The obtained sequence is compared with the original bit sequence to obtain the BER.

As an alternative to the two-times oversampled system, we consider also the case where the filter bank processing is done at the chip rate. Perfect code synchronization is assumed in both cases.

The frequency responses of the fourth subchannel filters that are used for the filter bank with $2 M=64$ complex channels are plotted in Figure 8. The figure shows a frequency modulated ELT prototype with overlapping factor $K=4$ and another more frequency selective one with $K=6$. In both cases, the roll-off in the filter bank design is $100 \%$, meaning that each subchannel transition band is overlapping with the closest transition band and passband of the adjacent subchannel, but not with the more distant ones.

Knowing the elements of this model, the number of affected and eliminated subbands can be estimated for each jammer power and a prediction for the expected performance based on (12) can be plotted. Depending on the stopband attenuation of the prototype filter in the suppressor, the number of affected subchannels varies with the power of 


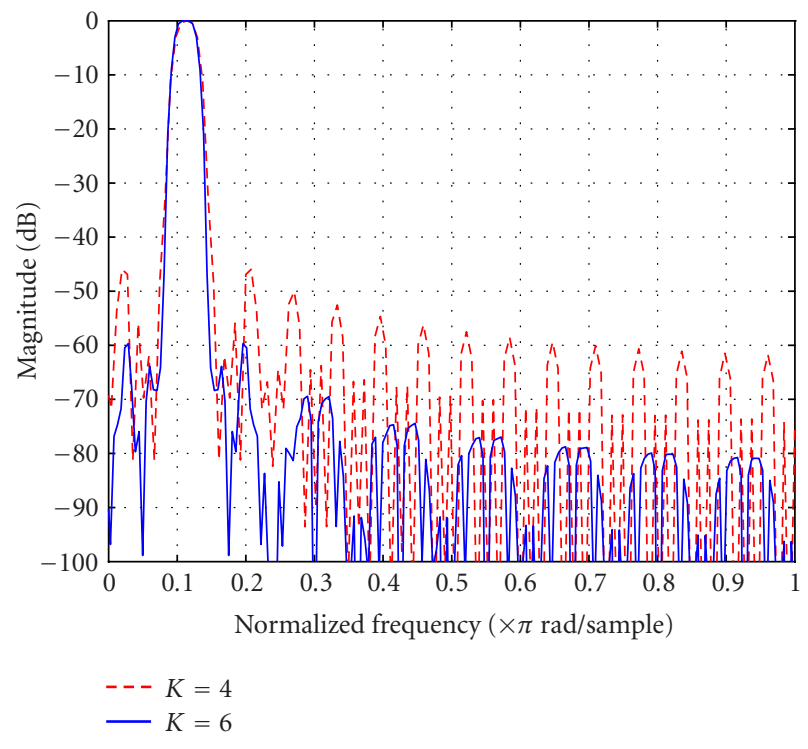

FIGURE 8: Frequency response for the analysis and synthesis banks of one of the subchannel filters with $K=4$ and $K=6$ where $2 M=$ 64 subbands.

the jammer. Thus, if the filter characteristics are known, the number of affected subbands can be predicted and the effective $S / N$ from (12) can be calculated to obtain the estimated BER for the two filter bank designs. An application of this idea is presented in Figure 13.

\section{PERFORMANCE EVALUATION}

Simulations with the previously described model were run with the following parameters: the spreading factor/processing gain of the spread spectrum system was $g_{p}=$ 127 and the spread signal was oversampled by 2 . The filter bank was an ELT-based complex bank with $2 M$ complex channels ( $M$ on the positive and $M$ on the negative sides of the frequency band), decimation, and interpolation by $M$ and perfect reconstruction. The threshold factor used in the jammer detection block was 2. For each point in the simulation results, at least 10000 data bits were used to check the BER. In the case of a randomly hopping jammer, the interferer hopped every 16 information bits and the simulations were done with $E_{b} / N_{0}=7 \mathrm{~dB}$. The results below extend the ones presented in [23].

Figure 9 compares the results applying the recursive algorithm with those that do not apply it with a $10 \%$ jammer and a single tone jammer at a fixed position. The improvement in the performance using the recursive algorithm is evident.

The previously mentioned effect of the transients is studied in Figure 10. The curves present the performance of a filter bank with $2 M=32$ subbands and with different prototype filters $K=4$ and $K=6$. It can be seen that without mitigating the transients, the results for both prototypes are very similar and we are not completely taking advantage of the higher stopband attenuation of the filter with higher overlapping factor $K$. However, when the guard interval method

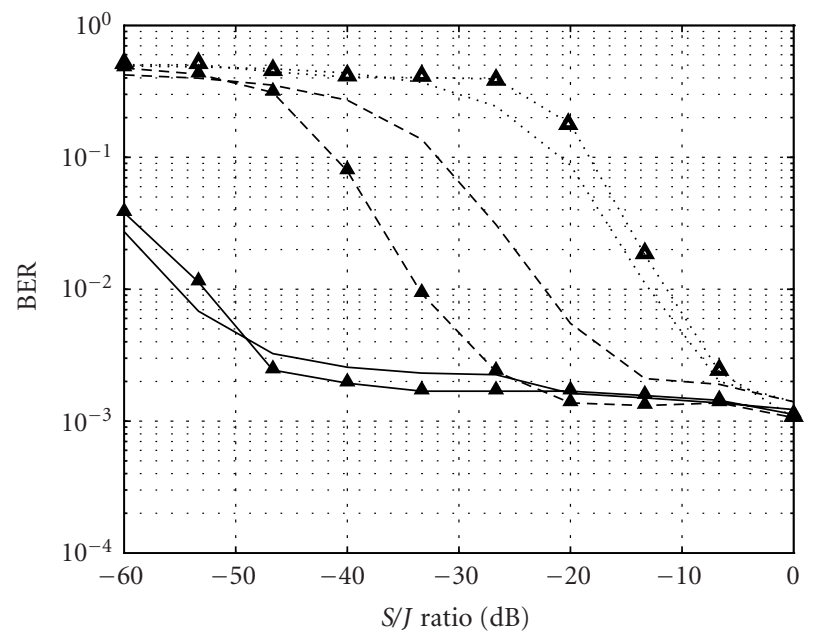

-. No excision, tone jammer

..... No excision, $10 \%$ jammer

- - One-time detection, $10 \%$ jammer

- $\Delta$ - One-time detection, tone jammer

- Recursive algorithm, 10\% jammer

$\neg$ Recursive algorithm, tone jammer

FIgure 9: Performance without excision, with one-time excision, and using the proposed recursive excision algorithm. $E_{b} / N_{0}=7 \mathrm{~dB}$, $2 M=32$ subbands with $K=4$, spreading factor $=127,10 \%$ and single tone jammers at fixed position. Guard-interval-based transient mitigation is applied.

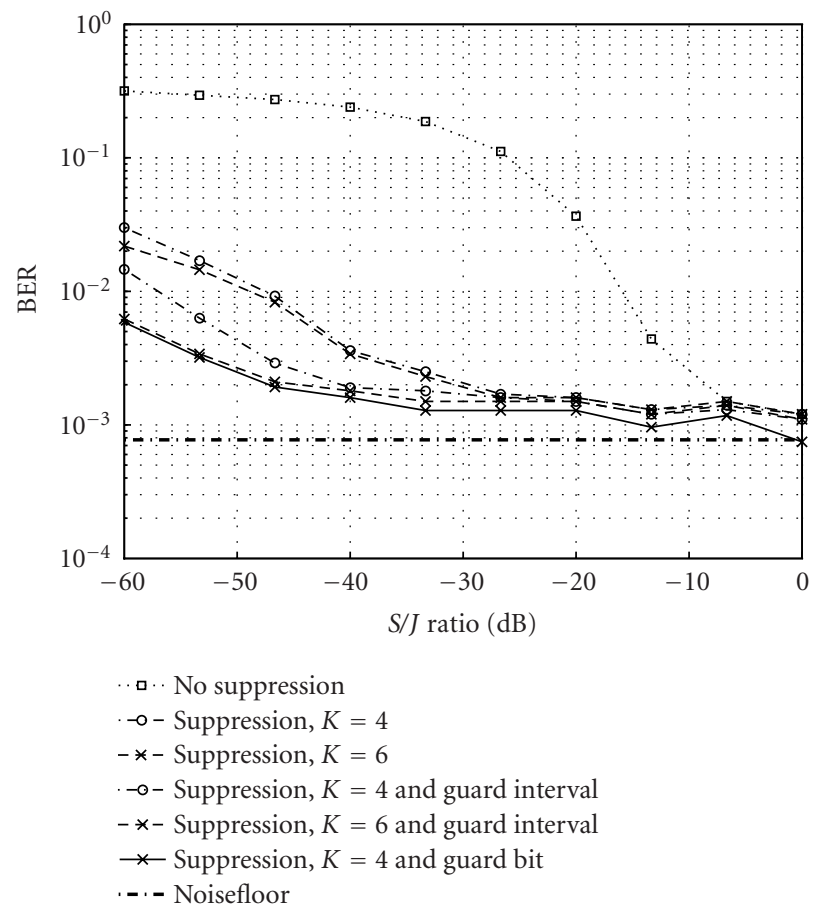

Figure 10: Fighting the transients with guard interval and guard bit. Filter prototypes with overlapping factors $K=4$ and $K=6$, where $E_{b} / N_{0}=7 \mathrm{~dB}, 2 M=32$ subbands, spreading factor $=127$, $10 \%$ jammer at randomly hopping positions. 


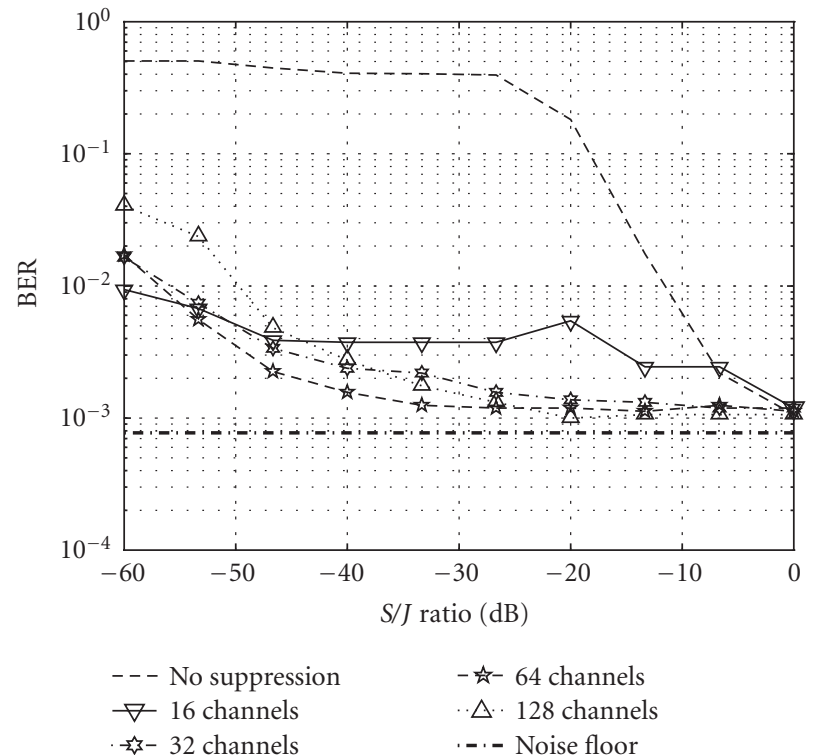

(a)

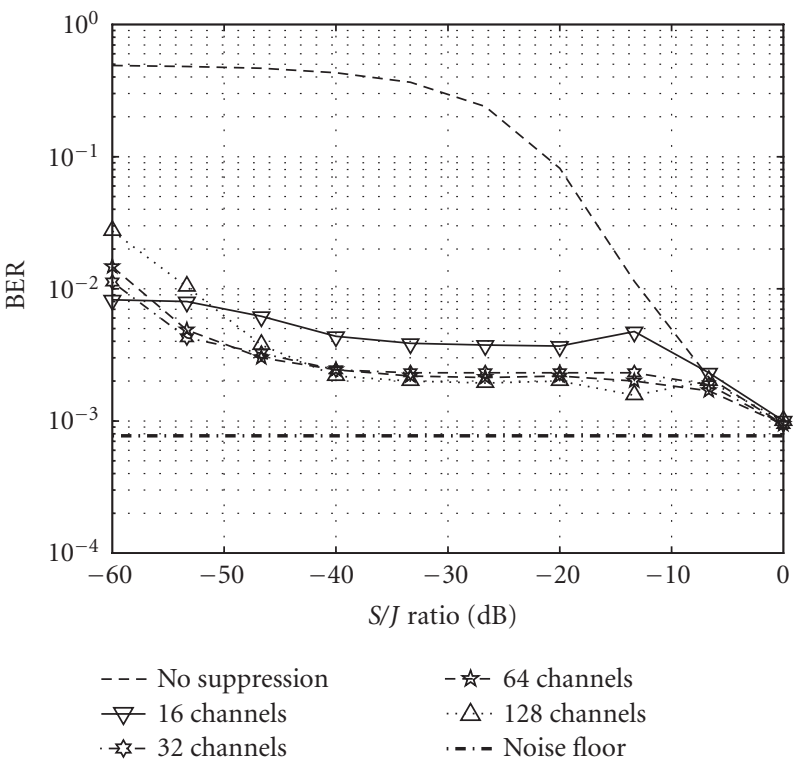

(c)

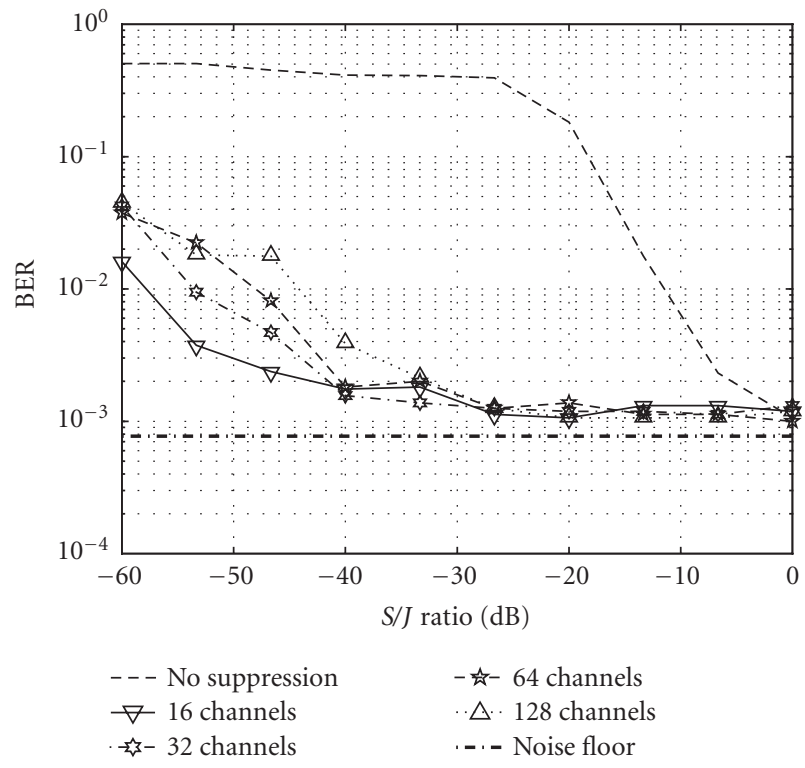

(b)

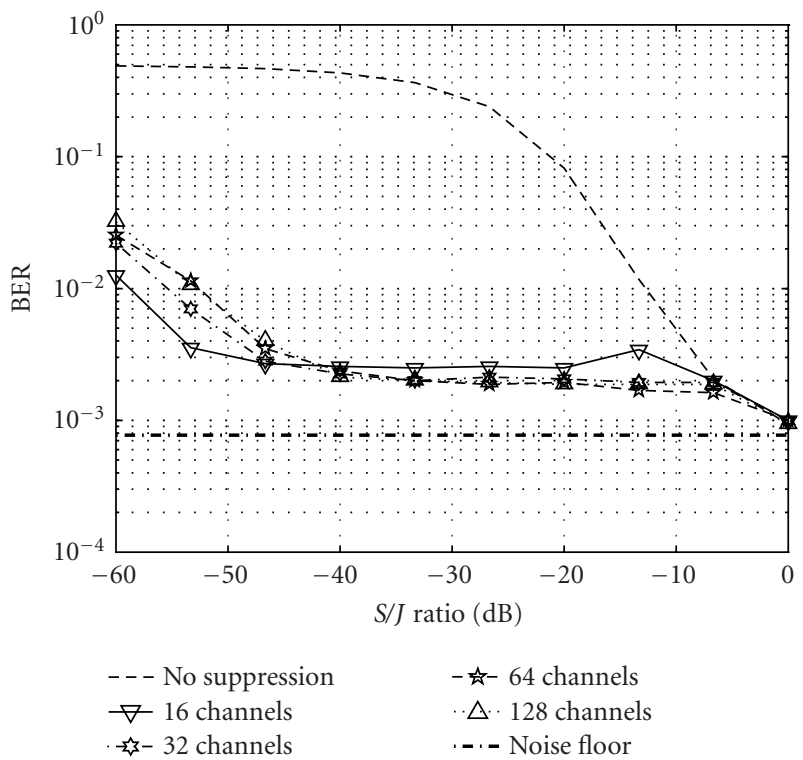

(d)

Figure 11: Performance with oversampled $(\mathrm{a}, \mathrm{c})$ and chip rate $(\mathrm{b}, \mathrm{d})$ processing using different filter bank sizes with $2 \mathrm{M}=16$ to 128 , where $K=6, E_{b} / N_{0}=7 \mathrm{~dB}$, spreading factor $=127$. ( $\left.\mathrm{a}, \mathrm{b}\right)$ represent the single tone and $(\mathrm{c}, \mathrm{d})$ represent the $10 \%$ jammers at fixed positions. Guard-interval-based transient mitigation is applied.

is applied, apart from an improvement in the performance in both designs, we can see that the difference between the performances grows. The figure also includes the guard bit approach as a lower BER bound for the guard interval method. With a guard interval of 20 chips, the performance of the guard interval idea is close to the performance of the guard bit approach. In the figure we also include the noise floor, representing the performance of the system when no jammer is present.
Several parameters have been modified during the simulations to investigate their effect on the performance of the system. For instance, the number of subchannels varied and the downsampling by two was performed before the filter bank (see the model in Figure 6), resulting in chip rate processing at the filter bank. Figures 11 and 12 combine the results of these variations. We can see that using $2 M=32$ gives a good performance with a reasonably low number of subbands. For the case $2 M=16$, the performance worsens, 


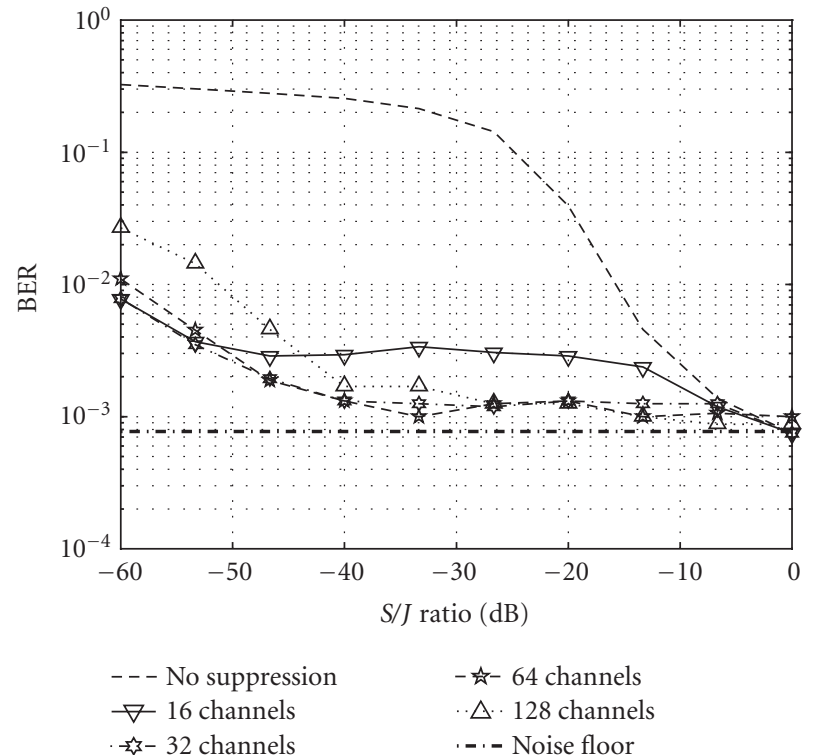

(a)

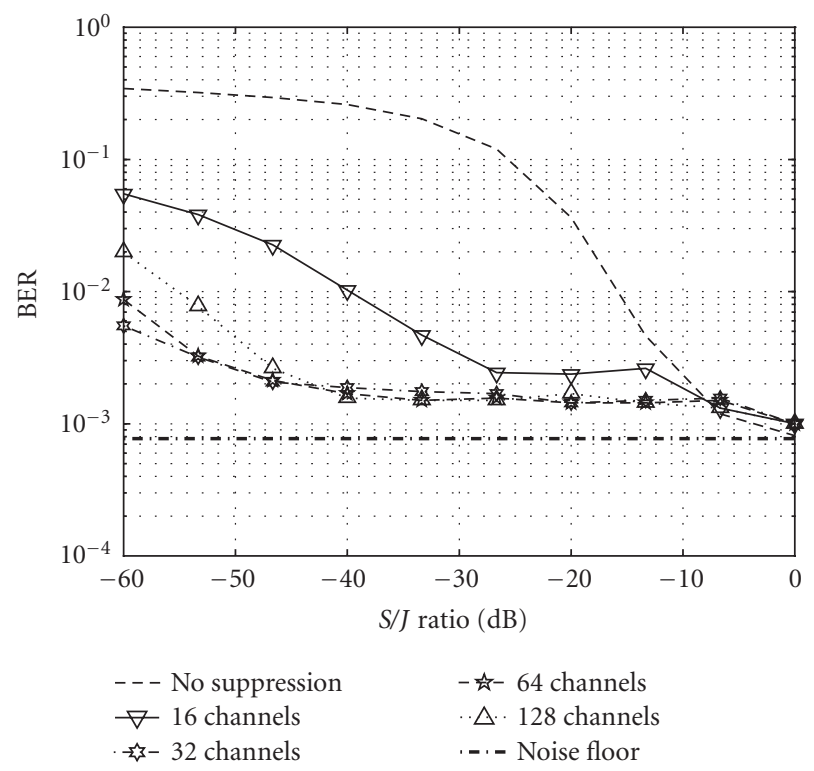

(c)

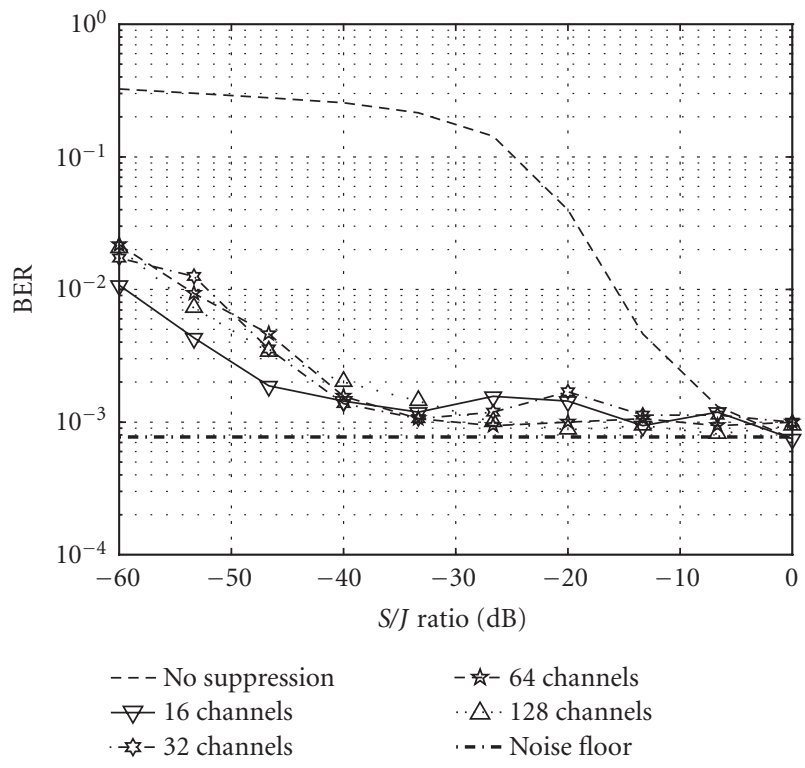

(b)

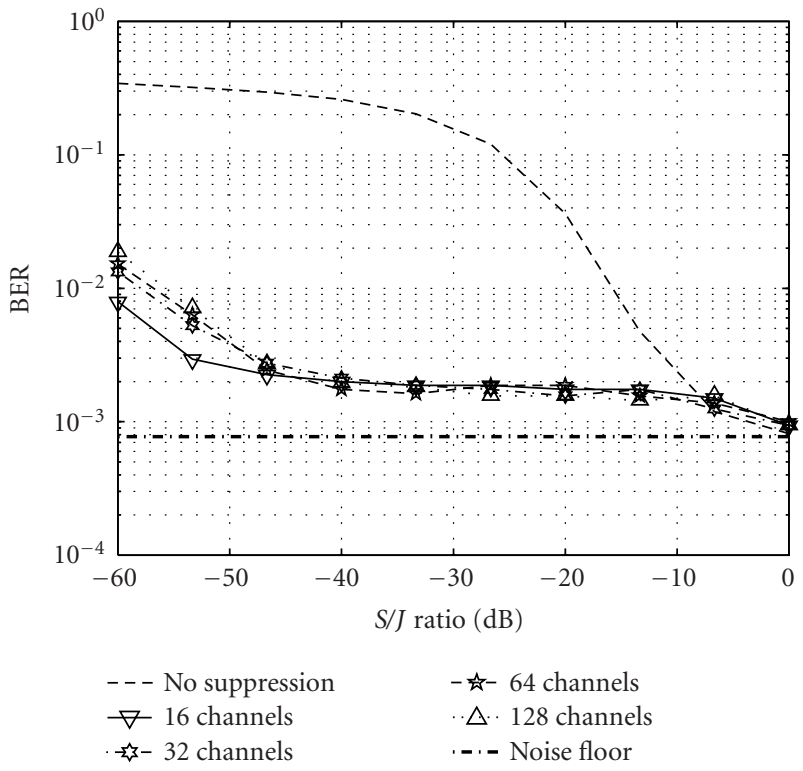

(d)

Figure 12: Performance with oversampled (a, c) and chip rate (b, d) processing using different filter bank sizes with $2 M=16$ to 128 , where $K=6, E_{b} / N_{0}=7 \mathrm{~dB}$, spreading factor $=127$. (a, b) represent the single tone and $(\mathrm{c}, \mathrm{d})$ represent the $10 \%$ jammers at randomly hopping positions. Guard-interval-based transient mitigation is applied.

and the difference with higher number of subchannels is especially high in the case of low-to-medium power jammers. Considering the case of chip rate processing, the width of the spectrum relative to the sampling frequency at the filter bank input is doubled, and this could allow having a filter bank with fewer subbands. The processing load can thus also be reduced in two ways, first by handling half the number of samples, second by using a smaller filter bank. In this case we see that also the filter bank with $2 M=16$ subbands achieves acceptable performance. Overall, using chip rate processing results only in a minor degradation in performance if the filter banks size is chosen appropriately.

The size of the filter bank affects the length of the prototype filter in its design: the larger $M$ is used, the more coefficients are needed for each subchannel filter. Longer subchannel filters cause also longer lasting transients, so a good trade-off between the length of the guard interval and the number of subbands in the bank has to be found. 


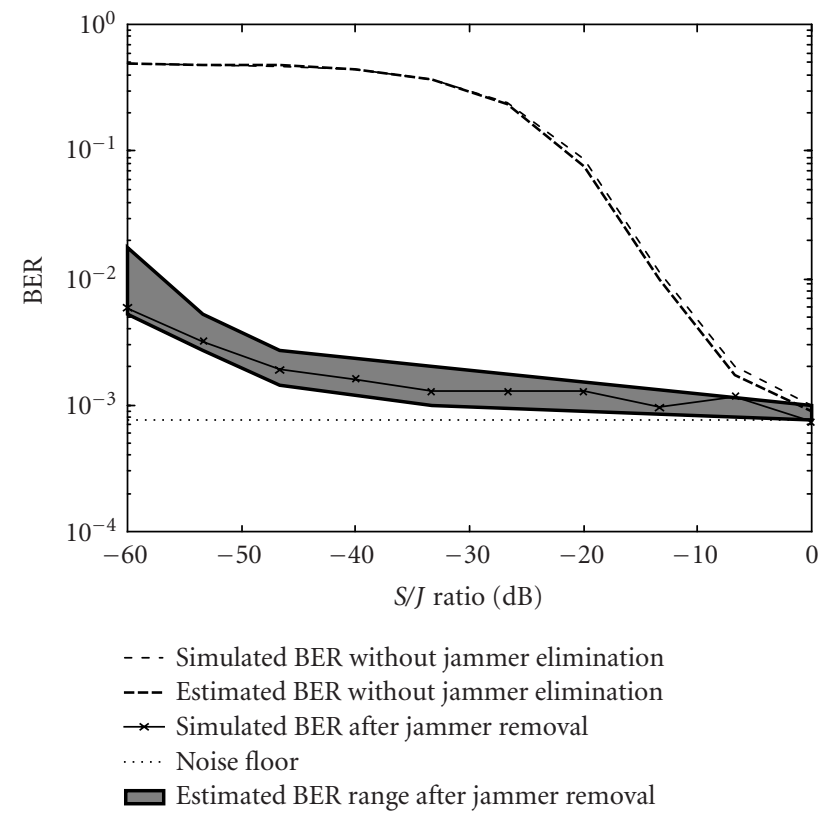

FIGURE 13: Expected BER range and obtained BER values over $S / J$ ratio in the optimised system with $2 M=32, K=6$, oversampled processing, guard bit, $E_{b} / N_{0}=7 \mathrm{~dB}$, spreading factor $=127$, different interference parameters.

We can compare the obtained results with the expected performance of the derivations of Section 3, taking into account the filter bank design proposed in Section 4. Figure 13 shows the estimated and simulated BER performances in the cases in which the jammer is not removed and also when it is removed. For the estimation of the BER range after the jammer removal, the range in the numbers of removed subbands at each $S / J$ ratio was considered. Knowing the type of interference and its power and the noise level, it is possible to predict how much the interference will stick out of the uniform DS-SS signal spectrum. Based on the stopband attenuation of the filters in the filter bank, we can then estimate how many subbands will be affected by the jammer, that is, get enough jammer energy to modify the uniformity of the desired signal. With the number of affected and therefore eliminated subbands and (12), we can calculate the expected degradation of the $E_{b} / N_{0}$ ratio and consequently the expected BER. Testing this idea on empirical measurements and counting the maximum and minimum number of affected subbands with different interference parameters, at each $S / J$ ratio, we were able to obtain the shaded area of Figure 13. The figure shows that the expected results match quite well with the obtained ones.

In another experiment, the processing was shortened into blocks of 2 to 8 information bits, instead of 16 as in the previous results. Shorter processing blocks permit the tracking and elimination of more quickly hopping jammers. The aim was to see how short the blocks could be made before the performance of the system decreased too much. Figures 14 and 15 reflect the results of this research. For a system of $K=6$, $2 M=128$ subbands at chip rate processing and using guard bits, the conclusion is that the degradation in performance is negligible for block lengths down to 6 bits, but beyond that point it starts to be significant.

The results shown in this section are clearly better than the ones presented in the reference method [8] using a 1024point FFT, as far as a direct comparison can be made. Apparently, the $10 \%$ BPSK jammer in [8] did not have any kind of pulse shaping, hence resulting in a more wideband signal with sinc spectrum. We present in Figure 16 a comparison between a filter bank with 32 subchannels and a 1024-point FFT (no windowing) under a 10\% fixed positioned jammer.

\section{CONCLUSIONS}

In this paper, a filter-bank-based interference detection and excision method for a DS-SS system has been studied and evaluated. The interfered subbands were removed from the signal to eliminate the jammers. The system worked with interference at a fixed position and with interference that randomly changed its position, with continuous wave interference and with BPSK type of interference taking up to $10 \%$ of the desired signal bandwidth.

The main strengths of the system presented in this paper are the perfect reconstruction property of the filter bank used and its affordable complexity requirements. It was shown through simulations that the performance is close to the theoretical limit when all aspects of the system are carefully optimised. The proposed system works quite well with far greater $S / J$ ratios than any of the transform domain techniques reported in the literature. We can take the results of [12, Figure $3.14]$ as a reference. They show the performance of different frequency domain excision methods in the case of $10 \%$ jammer bandwidth and indicate best performance for the ELT-based approach. In those results, the performance degrades drastically $(\mathrm{BER}>0.1$ ) for $S / J$ ratios lower than about $-45 \mathrm{~dB}$. For comparison, we repeated our simulations with similar parameters $\left(5 \mathrm{~dB} E_{b} / N_{0}\right.$, almost the same spreading code length, 63 instead of 64, but different spreading code). These results, with properly optimised filter bank and recursive jammer detection algorithm, indicate smoother degradation with low $S / J$ ratios providing tolerable performance $($ BER $<0.1)$ for $S / J$ above $-75 \mathrm{~dB}$ with $K=6(-55 \mathrm{~dB}$ with $K=4)$.

Implementing the perfect reconstruction filter bank with ELTs, an efficient system is obtained, allowing the system to work at high data rates. In [24], it was shown that the whole excision system with overlapping factor $K=5$ can be implemented with a single TMS320C6414 DSP with sampling rate in the order of $6-9 \mathrm{MHz}$, depending on the size of the filter bank.

One significant aspect when comparing with most of the other frequency domain approaches is that the needed number of subchannels is very low. Even with 16 subchannels, the performance is close to the theoretical one.

All in one, the narrowband interference suppression method presented is a good compromise between complexity, efficiency, and performance at relatively high jammer powers. In [9], a similar conclusion is drawn when 

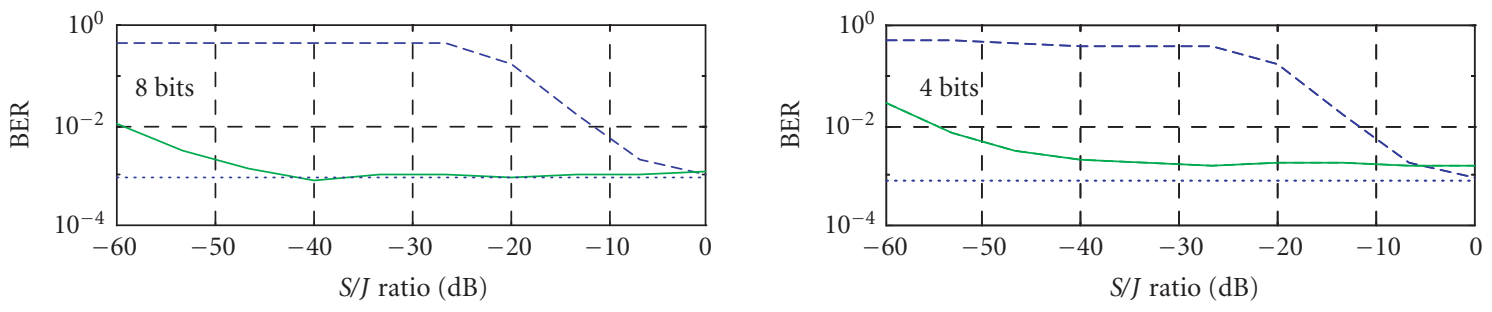

(a)
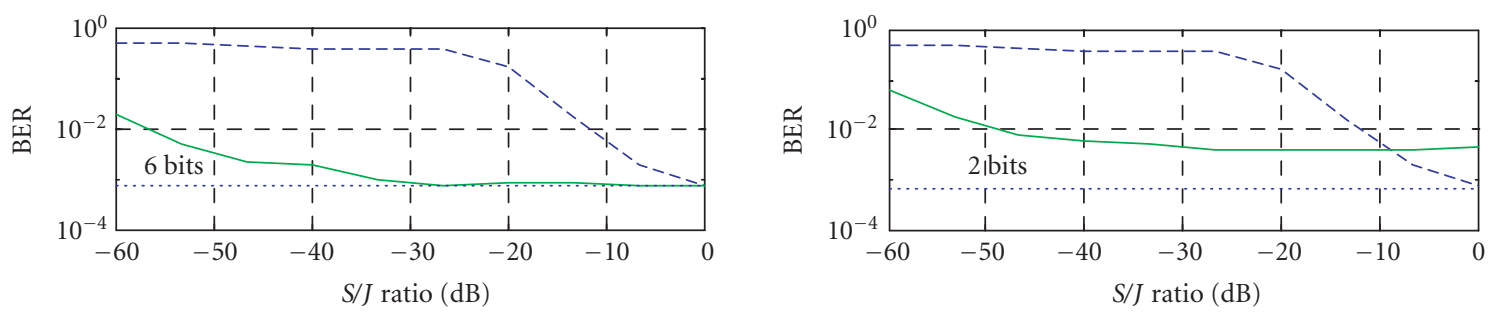

(b)
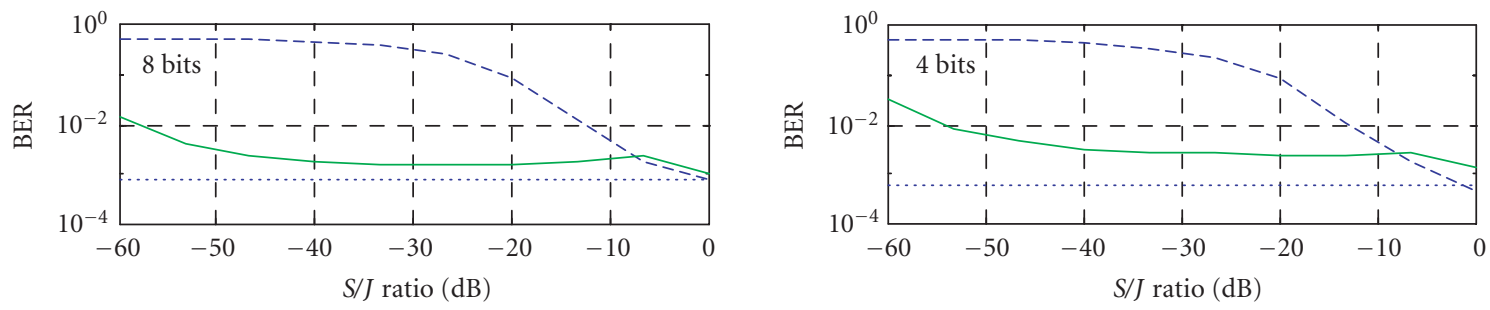

(c)
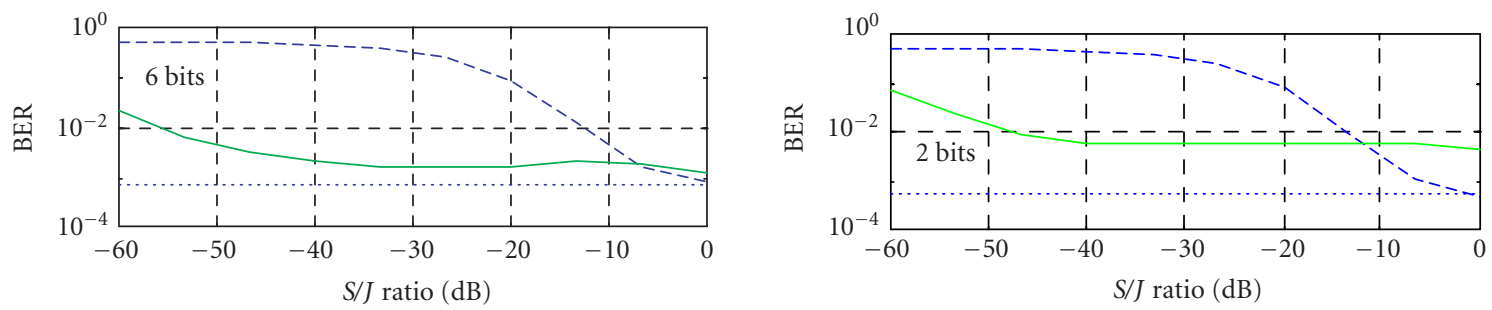

(d)

FIGURE 14: Different processing block lengths of 2 to 8 bits. Filter bank with $2 M=128, K=6$, chip rate processing, guard bit, $E_{b} / N_{0}=7 \mathrm{~dB}$, and spreading factor $=127$. (a), (b) represent the single tone and (c), (d) represent the 10\% jammers at fixed positions. Dashed lines represent simulated BER without jammer removal, solid lines represent simulated BER after jammer removal, and dotted lines represent noise floor.

comparing ELT, FFT, and DCT methods. Better performance at increasing jammer powers can be obtained by using prototype filters that are more frequency selective and have higher stopband attenuation. However, the limiting factors can be at earlier stages in the receiver. For example, the resolution of the $\mathrm{A} / \mathrm{D}$ converter has to be good enough to represent a desired signal that is, for instance, $70 \mathrm{~dB}$ weaker than the interfering signal that is also being sampled simultaneously.

We have studied the performance of the proposed method only in the single-user case. In multiuser CDMA en- vironment, the effect of the notch filtering due to filter bank excision on the multiple access interference (MAI) is a serious concern. Considering the case of very strong narrowband interference, it is obvious that the best thing to do also from the MAI point of view is to remove the interfered frequency band completely since it could not include any useful information for detection. However, in this context, the use of higher number of subchannels than needed in the singleuser case could be favourable. In case of modest jammer levels, subband excision is clearly a suboptimal solution and 

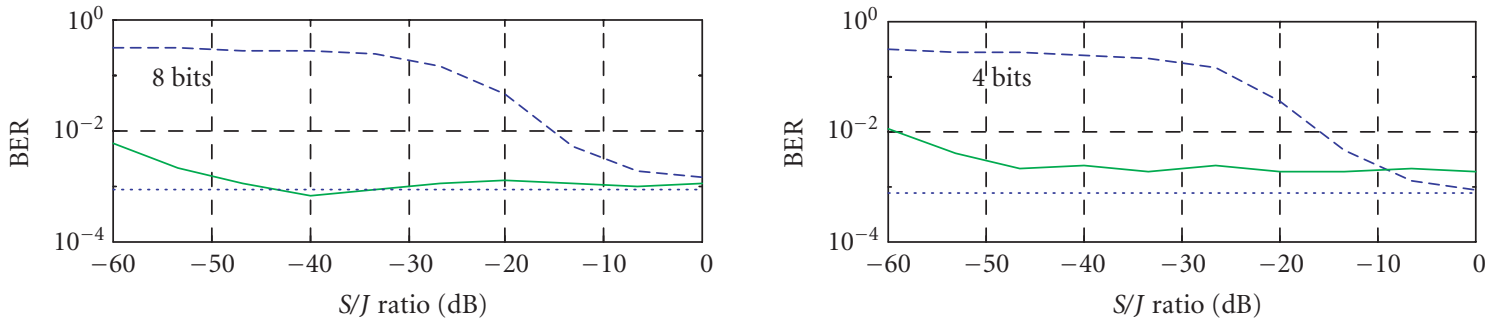

(a)
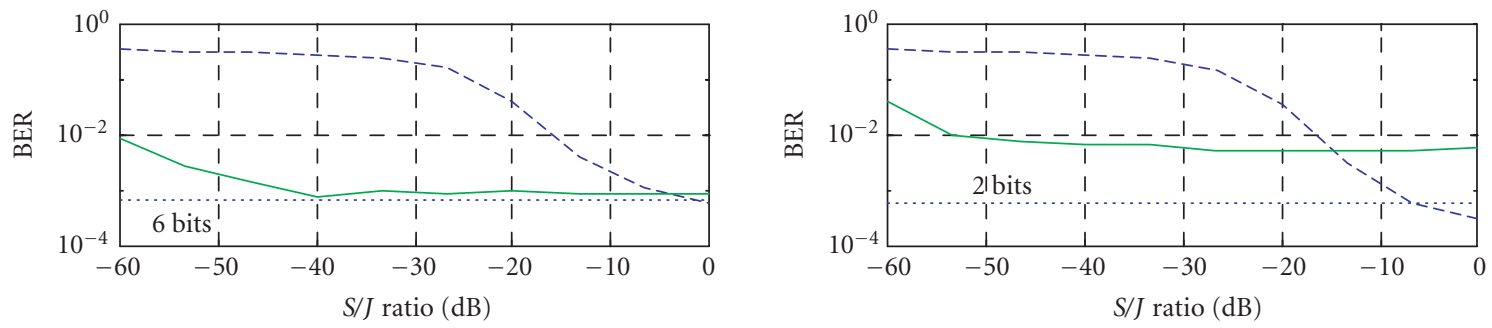

(b)
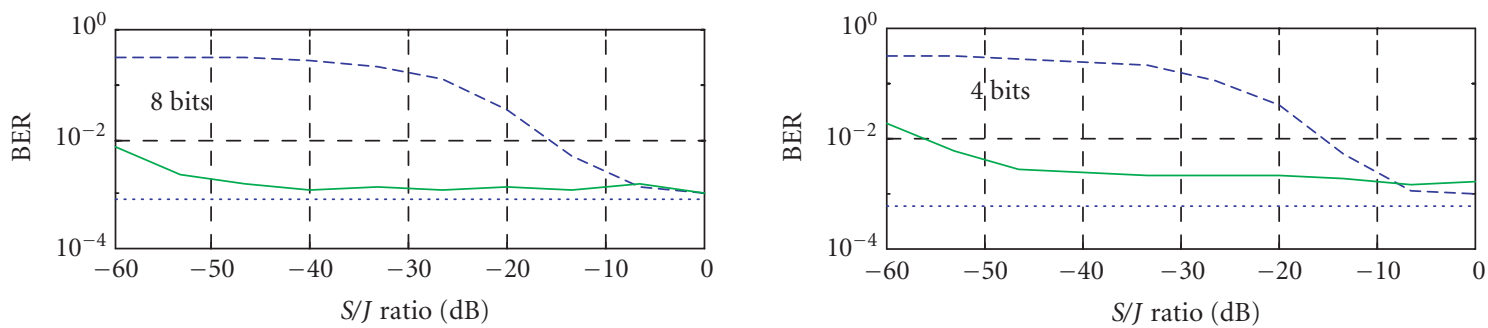

(c)
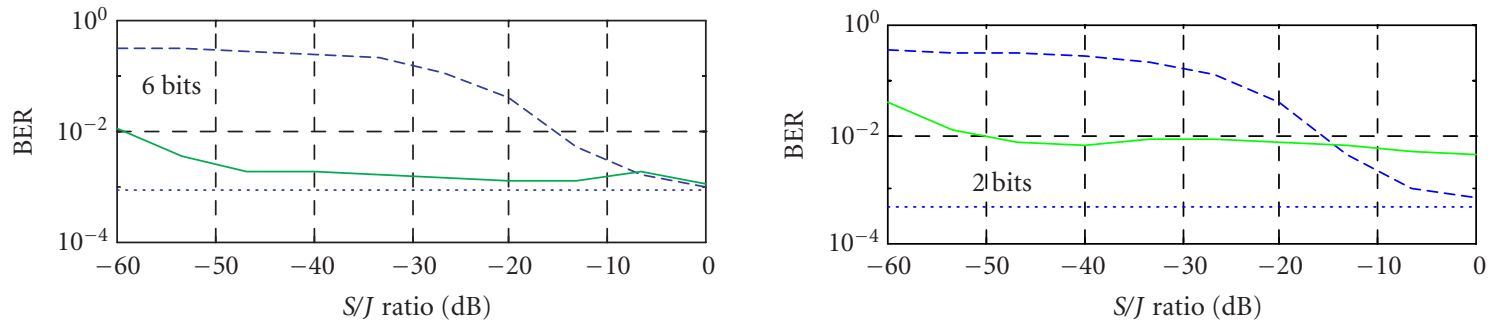

(d)

Figure 15: Different processing block lengths of 2 to 8 bits. Filter bank with $2 M=128, K=6$, chip rate processing, guard bit, $E_{b} / N_{0}=7 \mathrm{~dB}$, and spreading factor $=127$. (a), (b) represent the single tone and (c), (d) represent the $10 \%$ jammers at randomly hopping positions. Dashed lines represent simulated BER without jammer removal, solid lines represent simulated BER after jammer removal, and dotted lines represent noise floor.

its performance comparisons against other interference suppression methods are a topic for future studies. Also a more analytical study of the recursive jammer detection methods is included in the future plans.

\section{ACKNOWLEDGMENT}

This work was carried out in the project "Digital and Analog Techniques in Flexible Receivers" funded by the National Technology Agency of Finland (Tekes). 


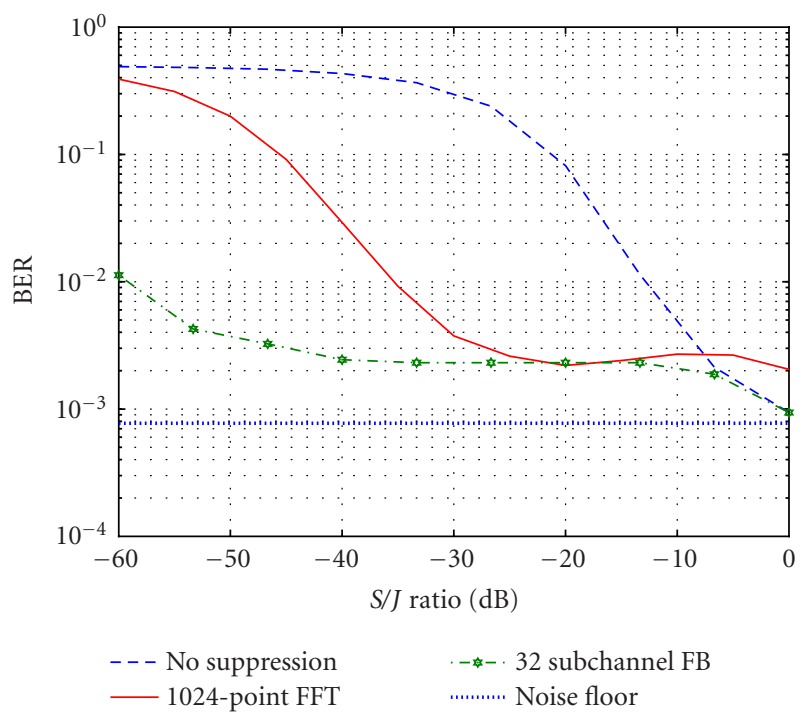

FIgURE 16: Comparison between a filter bank with $2 M=32$, $K=6$ and oversampled processing and a 1024-point FFT, where $E_{b} / N_{0}=7 \mathrm{~dB}$, spreading factor $=127$ and the $10 \%$ jammer is at a fixed position. Guard-interval-based transient mitigation is applied.

\section{REFERENCES}

[1] J. G. Proakis, Digital Communications, Electrical Engineering Series. McGraw-Hill International Editions, New York, NY, USA, 4th edition, 2001.

[2] L. B. Milstein, "Interference rejection techniques in spread spectrum communications," Proceedings of the IEEE, vol. 76, no. 6, pp. 657-671, 1988.

[3] F. Hsu and A. Giordano, "Digital whitening techniques for improving spread spectrum communications performance in the presence of narrowband jamming and interference," IEEE Trans. Communications, vol. 26, no. 2, pp. 209-216, 1978.

[4] J. W. Ketchum and J. G. Proakis, "Adaptive algorithms for estimating and suppressing narrow-band interference in PN spread-spectrum systems," IEEE Trans. Communications, vol. 30, no. 5, pp. 913-924, 1982.

[5] R. A. Iltis and L. B. Milstein, "Performance analysis of narrowband interference rejection techniques in DS spread-spectrum systems," IEEE Trans. Communications, vol. 32, no. 11, pp. 1169-1177, 1984.

[6] P. Henttu, H. Saarnisaari, and S. Aroinaa, "Interference suppression in DS/FH system using modified two sided adaptive filter," in Proc. IEEE Military Communications Conference, pp. 1429-1433, McLean, Va, USA, October 2001.

[7] M. J. Medley, G. J. Saulnier, and P. K. Das, "Narrow-band interference excision in spread spectrum systems using lapped transforms," IEEE Trans. Communications, vol. 45, no. 11, pp. 1444-1455, 1997.

[8] A. Pouttu, J. K. Juntti, and T. J. Kumpumäki, "Adaptive transform domain interference suppression in a hybrid DS/FHsystem," in Proc. IEEE 5th International Symposium on Spread Spectrum Techniques and Applications, pp. 351-355, Sun City, South Africa, September 1998.

[9] P. Henttu, A. Pouttu, and M. Raustia, "Performance of PIE interference suppressor using FFT, DCT and ELT transformations in FH/DS communications," in Proc. Information Systems for Enhanced Public Safety and Security. IEEE/AFCEA, pp. 126-130, Munich, Germany, May 2000.
[10] R. A. Monzingo and T. W. Miller, Introduction to Adaptive Arrays, John Wiley \& Sons, New York, NY, USA, 1980.

[11] M. V. Tazebay and A. N. Akansu, "Adaptive subband transforms in time-frequency excisers for DSSS communications systems," IEEE Trans. Signal Processing, vol. 43, no. 11, pp. 2776-2782, 1995.

[12] A. N. Akansu and M. J. Medley, Wavelet, Subband, and Block Transforms in Communications and Multimedia, Kluwer Academic, Boston, Mass, USA, 1999.

[13] P. P. Vaidyanathan, Multirate Systems and Filter Banks, Prentice-Hall Signal Processing Series. Prentice-Hall, Englewood Cliffs, NJ, USA, 1993.

[14] T. J. Kumpumäki, M. A. Isohookana, and J. K. Juntti, "Narrow-band interference rejection using transform domain signal processing in a hybrid DS/FH spread spectrum system," in Proc. IEEE Military Communications Conference, pp. 89-93, Monterey, Calif, USA, November 1997.

[15] T. Karp and N. J. Fliege, "Modified DFT filter banks with perfect reconstruction," IEEE Trans. on Circuits and Systems II: Analog and Digital Signal Processing, vol. 46, no. 11, pp. 1404-1414, 1999.

[16] A. Viholainen, T. H. Stitz, J. Alhava, T. Ihalainen, and M. Renfors, "Complex modulated critically sampled filter banks based on cosine and sine modulation," in Proc. IEEE Int. Symp. Circuits and Systems, pp. 833-836, Scottsdale, Ariz, USA, May 2002.

[17] H. S. Malvar, Signal Processing with Lapped Transforms, Artech House, Boston, Mass, USA, 1992.

[18] J. Alhava, A. Viholainen, and M. Renfors, "Efficient implementation of complex exponentially-modulated filter banks," in Proc. IEEE Int. Symp. Circuits and Systems, pp. 157-160, Bangkok, Thailand, May 2003.

[19] H. S. Malvar, "A modulated complex lapped transform and its applications to audio processing," in Proc. IEEE Int. Conf. Acoustics, Speech, Signal Processing, pp. 1421-1424, Phoenix, Ariz, USA, March 1999.

[20] R. W. Young and N. G. Kingsbury, "Frequency-domain motion estimation using a complex lapped transform," IEEE Trans. Image Processing, vol. 2, no. 1, pp. 2-17, 1993.

[21] A. Viholainen, J. Alhava, and M. Renfors, "Implementation of parallel cosine and sine modulated filter banks for equalized transmultiplexer systems," in Proc. IEEE Int. Conf. Acoustics, Speech, Signal Processing, pp. 3625-3628, Salt Lake City, Utah, USA, May 2001.

[22] P. Henttu, "Signaalikomponenttien erottaminen radiojärjestelmän vastaanottimessa," in Suomalainen Pattentihakemus nro. 20010952, Nokia Networks Oy, Finland, 2001.

[23] T. H. Stitz and M. Renfors, "Filter bank based narrowband interference detection and suppression in spread spectrum systems," in Proc. IEEE Int. Symp. Circuits and Systems, pp. 516519, Phoenix-Scottsdale, Ariz, USA, May 2002.

[24] Y. Yang, T. H. Stitz, and M. Renfors, "Implementation of a filter bank based narrowband interference suppression algorithm on a DSP processor," in Proc. International Conference on Telecommunications, Beijing, China, June 2002.

Tobias Hidalgo Stitz was born in 1974 in Eschwege, Germany. He obtained the M.S. degree in telecommunications engineering from the Polytechnic University of Madrid (UPM) in 2001, after writing his Master's thesis at the Institute of Communications Engineering of the Tampere University of Technology (TUT). From 1999 to 2001, he was a Research Assistant at TUT and is now

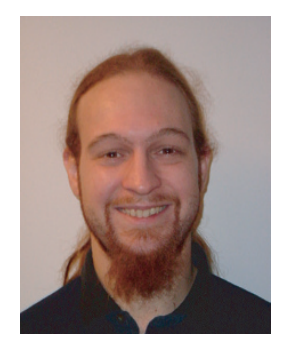


working towards his doctoral degree there. His research interests include wireless communications based on multicarrier systems, especially focusing on filter-bank-based systems and other filter bank applications for signal processing.

Markku Renfors was born in Suoniemi, Finland, on January 21, 1953. He received the Diploma Engineer, Licentiate of Technology, and Doctor of Technology degrees from Tampere University of Technology (TUT) in 1978, 1981, and 1982, respectively. He held various research and teaching positions at TUT from 1976 to 1988 . In the years 1988-1991, he was working as a Design Manager in the area of video signal pro-

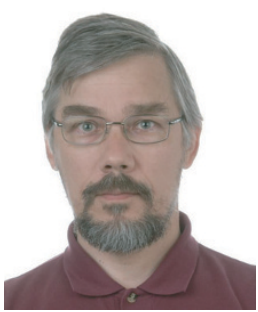
cessing, especially for HDTV, at Nokia Research Centre and Nokia Consumer Electronics. Since 1992, he has been a Professor and Head of the Institute of Communications Engineering at TUT. His main research areas are multicarrier systems and signal processing algorithms for flexible radio receivers and transmitters. 